\title{
Diffusion-Weighted MR Imaging of the Liver at 3.0 Tesla Using Tracking Only Navigator Echo (TRON): a Feasibility Study
}

\author{
Marko K. Ivancevic, PhD, ${ }^{1,2 *}$ Thomas C. Kwee, MD, ${ }^{2,3}$ Taro Takahara, MD, PhD, ${ }^{3}$ \\ Tetsuo Ogino, MSc, ${ }^{4}$ Hero K. Hussain, MD, ${ }^{2}$ Peter S. Liu, MD, ${ }^{2}$ \\ and Thomas L. Chenevert, PhD $^{2}$
}

Purpose: To assess the feasibility of TRacking Only Navigator echo (TRON) for diffusion-weighted magnetic resonance imaging (DWI) of the liver at 3.0T.

Materials and Methods: Ten volunteers underwent TRON, respiratory triggered, and free breathing DWI of the liver at 3.0 Tesla (T). Scan times were measured. Image sharpness, degree of stair-step and stripe artifacts for the three methods were assessed by two observers.

Results: Mean scan times of TRON and respiratory triggered DWI relative to free breathing DWI were 34\% and $145 \%$ longer respectively. In four of eight comparisons (two observers, two b-values, two slice orientations), TRON DWI image sharpness was significantly better than free breathing DWI, but inferior to respiratory triggered DWI. In two of four comparisons (two observers, two b-values), degree of stair-step artifacts in TRON DWI was significantly lower than in respiratory triggered DWI. Degree of stripe artifacts between the three methods was not significantly different.

Conclusion: DWI of the liver at 3.OT using TRON is feasible. Image sharpness in TRON DWI is superior to that in free breathing DWI. Although image sharpness of respiratory triggered DWI is still better, TRON DWI requires less scan time and reduces stair-step artifacts.

Key Words: diffusion-weighted magnetic resonance imaging; liver; tracking only navigator echo; TRON; high field strength; 3.0T

J. Magn. Reson. Imaging 2009;30:1027-1033.

(C) 2009 Wiley-Liss, Inc.

\footnotetext{
${ }^{1}$ Philips Healthcare, Cleveland, Ohio, USA.

${ }^{2}$ University of Michigan, Department of Radiology, Ann Arbor, Michigan, USA.

${ }^{3}$ University Medical Center Utrecht, Department of Radiology, Utrecht, The Netherlands.

${ }^{4}$ Philips Electronics Japan, Ltd, Yokohama, Japan.

*Address reprint requests to: M.K.I., Philips Healthcare, University of Michigan, Department of Radiology, MRI, 1500 East Medical Center Drive, Ann Arbor, MI, 48109. E-mail: marko.ivancevic@philips.com Received May 22, 2009; Accepted August 7, 2009.

DOI $10.1002 /$ jmri.21939

Published online in Wiley InterScience (www.interscience.wiley.com).
}

DIFFUSION-WEIGHTED MAGNETIC resonance (MR) imaging (DWI) is an emerging technique for the detection and characterization of liver lesions (1). Two types of non-breathhold DWI are used in clinical practice: respiratory gated (either using a conventional navigator echo technique or respiratory triggering) and free breathing DWI (with multiple signal averaging). Advantages of non-breathhold techniques compared with breathhold DWI are the possibility to increase signal-to-noise ratio (SNR) by acquiring multiple signal averages, which allows obtaining thin slices. This, in turn, may increase lesion detection rate. However, respiratory gated DWI suffers from a prolonged examination time, while free breathing DWI may suffer from image blurring $(1,2)$. Recently, the limitations of both respiratory gated and free breathing DWI have been overcome by the introduction of the TRacking Only Navigator echo (TRON) technique (2-4), a self-implemented sequence extension. TRON uses a navigator echo for continuous real-time slice tracking and position correction, without use of any gating window. Consequently, scan time is not prolonged and image blurring is reduced (2-4).

A general disadvantage of DWI is its relative low SNR. Nevertheless, SNR can be increased when performing DWI at higher field strength (3.0 Tesla [T]) (5). Combining the advantages of DWI at 3.0T with TRON may be of particular benefit. However, besides the stronger susceptibility effects at 3.0T that may degrade image quality itself (5), implementation of TRON at 3.0T is also challenging. First, field inhomogeneity may affect the quality of the navigator echo data. While 3.0T suffers from higher BO and B1 field inhomogeneities, B1 typically varies over a large field of view. For the limited location of navigator-diaphragm interface BO inhomogeneity will be the most critical. Second, T1 is significantly longer at 3.0T compared with $1.5 \mathrm{~T}$ (5). Consequently, a navigator echo at 3.0T may yield reduced signal in case of incomplete T1 recovery or saturation of the actual image volume that overlaps with the navigator echo. As a result, the navigator profile may not depict the true position of the liver, which may affect tracking accuracy. Despite 


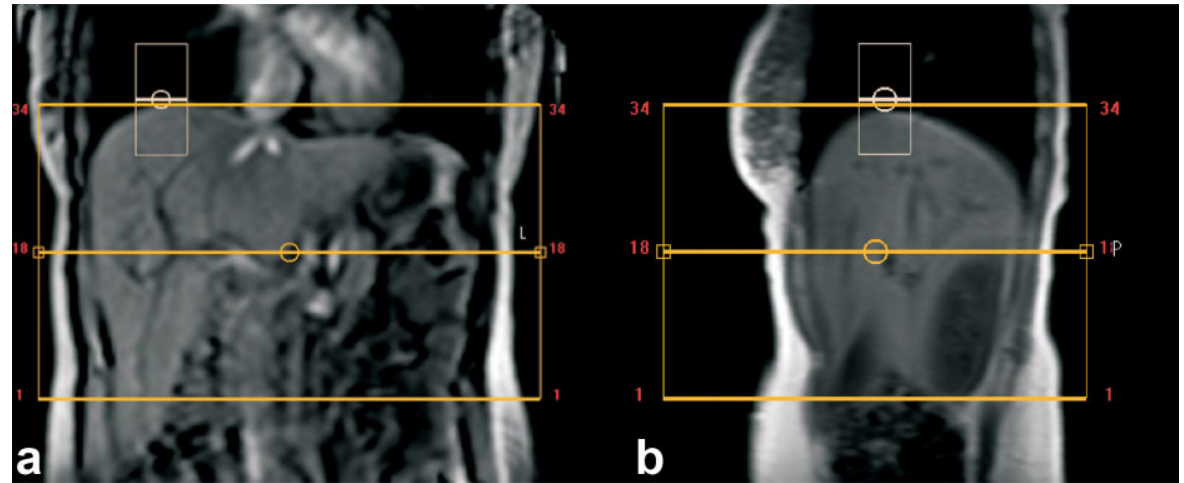

Figure 1. Coronal (a), and sagittal (b) survey images showing placement of the pencil-beam excitation prepulse (navigator echo) that was used for tracking and position correction in TRON, at the interface between liver and lung. [Color figure can be viewed in the online issue, which is available at www. interscience.wiley.com.] these challenges, this study aimed to assess the feasibility of the TRacking Only Navigator echo (TRON) technique for DWI of the liver at 3.0T.

\section{MATERIALS AND METHODS}

\section{Study Participants}

This study was approved by the local Institutional Review Board and written informed consent was obtained from all participants. Ten healthy volunteers (6 men and 4 women; mean age, 31.7 years; age range, 23-55 years) prospectively underwent DWI of the liver. Exclusion criteria were general contraindications to MR imaging.

\section{MR Imaging}

MR imaging was performed using a whole-body 3.0T system (Achieva, Philips Healthcare, Best, The Netherlands), with a 16-element phased array surface coil for signal reception. For all volunteers, three types of DWI of the liver were performed: TRON, respiratory triggered, and free breathing DWI. TRON is a modified navigator echo technique. Similar to the conventional navigator echo technique, a pencil-beam excitation prepulse was placed at the interface between liver and lung (Fig. 1). The diaphragm position for each time point is determined by cross correlation between a reference kernel (expiration position) and the actual navigator beam signal. The transmit frequency of the rf pulse is adjusted to displace the slice according to the position shift determined from the navigator signal. Flip angle of the navigator echo was $25^{\circ}$. A fat saturation pulse was applied following the navigator readout, and time delay between the navigator echo and the next slice excitation was $32 \mathrm{~ms}$. Slices were acquired in a descending interleaved order. Two slices of $92 \mathrm{~ms}$ sampling duration were acquired subsequent to each navigator readout.

However, unlike the conventional navigator echo technique, the navigator echo in TRON is only used to track and correct for tissue displacement, and is not used for gating. That is, the actual slice locations are updated in real-time based on tracking of diaphragm position. To mitigate signal loss on the navigator due to possible $\mathrm{T} 1$ saturation effects, anomalous dark bands from previous slice excitations are detected in the navigator and corrected by linear interpolation before calculation of positional change. Thus unlike conventional navigator-based gating, with TRON, data can be acquired during the entire respiratory cycle (Fig. 2). It was anticipated that the transition from $1.5 \mathrm{~T}$ to $3.0 \mathrm{~T}$ could affect the quality of the navigator echo and reduce tracking accuracy due to BO inhomogeneities and $\mathrm{T} 1$ saturation effects of the slices that overlap with the navigator echo beam (5) as seen in Figure 3. Liver motion in the superior-inferior direction is presumed to not exceed a certain distance between two consecutive navigator echoes. However, if the quality of the navigator echo is imperfect, overestimation of the true liver displacement may occur, leading to slice misregistration. Therefore, the original TRON technique was slightly modified, in that data were not accepted if the navigator echo detected a displacement (relative to the previous navigator echo) that exceeded a specified threshold, called "maximum displacement" (Figs. 2, 3). In this study, maximum displacement was set to $10 \mathrm{~mm}$. For respiratory triggered DWI, a respiratory pressure sensor was fixed to the abdominal region by an elastic belt, and an endexpiratory trigger delay of $250 \mathrm{~ms}$ was applied.

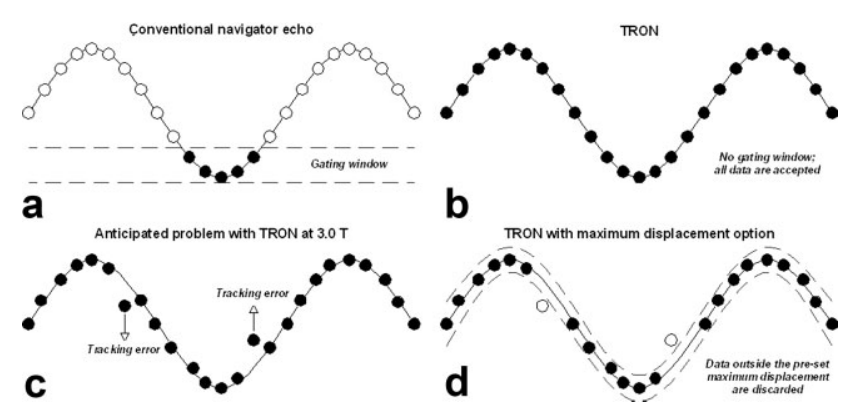

Figure 2. Concepts of the conventional navigator echo technique (a), TRON (b), anticipated problem when using TRON at 3.OT (c), and TRON with maximum displacement option (d). Using the conventional navigator echo technique, the timing of the acquisition is adjusted so that data are acquired only during a specific range of diaphragmatic motion. In TRON, there is no gating window, and all data are accepted (b). The quality of the navigator echo may be imperfect at 3.0T, which may lead to tracking errors and slice misregistration (c). The original TRON technique was slightly modified, in that data were not accepted if the navigator echo detected a displacement (relative to the previous navigator echo) that exceeded a certain threshold (maximum displacement) (d). 


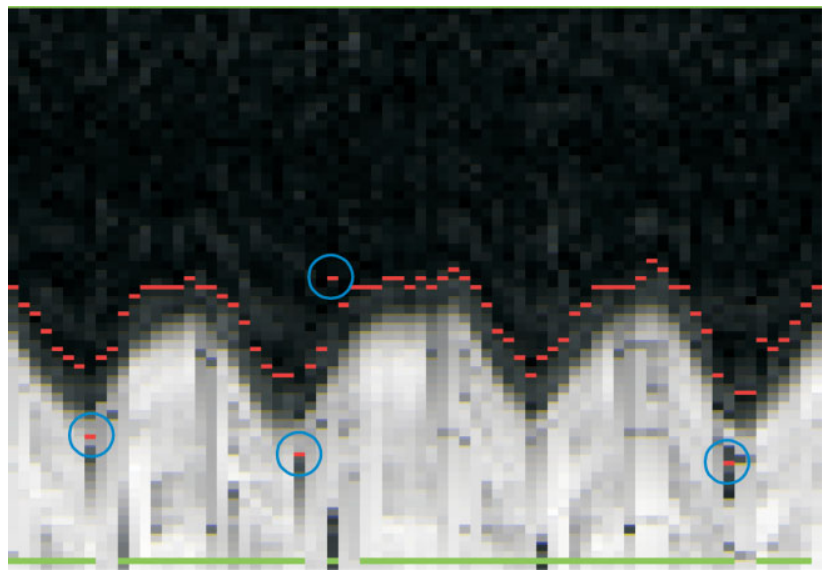

Figure 3. Representative example of a part of a navigator profile in TRON using the maximum displacement limit of $10 \mathrm{~mm}$. The navigator echo profile shows the diaphragm motion, indicated by the white wave. Red dots represent tracking of the diaphragmatic position using navigator echoes. In this part of the navigator profile, 75 navigator echoes were acquired. T1 saturation causes dark bands in the navigator profile. Although visually the border between lung and tissue is apparent, the dark band causes a miscalculation of the diaphragm position. Therefore, by limiting maximum displacement to $10 \mathrm{~mm}$, the acquisition for these four erroneous navigator points is skipped (encircled).

DWI was performed with the following parameters for TRON (Table 1): single-shot spin-echo echo-planar imaging (EPI), repetition time (TR)/echo time (TE) of $4294 / 58 \mathrm{~ms}$, image acquisition in the axial plane, slice thickness/gap of $5 / 0 \mathrm{~mm}$, number of slices of 34 , field of view $(\mathrm{RL} \times \mathrm{AP})$ of $280-250 \times 245-330 \mathrm{~mm}$, motion probing gradients (MPG) in three orthogonal axes, b-values of 0,50 , and $500 \mathrm{~s} / \mathrm{mm}^{2}$, number of signal averages (NSA) of 4 , half scan factor of 0.6 , parallel acceleration (SENSitivity Encoding, SENSE) factor of 2, EPI factor of 53-69, spectral presaturation inversion recovery (SPIR) fat suppression, acquired voxel size of $2.50 \times 2.5 \times 5.00 \mathrm{~mm}$, reconstructed voxel size of $1.25 \times 1.25 \times 5.00 \mathrm{~mm}$, and nominal scan time of $2 \mathrm{~min}$ and 3-6 s. With voxel size kept constant matrix varied accordingly to the FOV: 100$112 \times 98-130$. Image acquisition parameters for respiratory triggered DWI were identical, except for a TR that matched the subject's respiratory cycle, and a nominal scan time of $1 \mathrm{~min}$ and $45-48 \mathrm{~s}$. Image acquisition parameters for free breathing DWI were also identical, except for a TR of $3691 \mathrm{~ms}$ and a nominal scan time of $1 \mathrm{~min}$ and 45-48 $\mathrm{s}$ (depending on FOV adjustments).

Actual scan times of TRON and respiratory triggered DWI were manually recorded for each subject. All axial images were coronally reformatted, with a slice thickness/gap of $5 / 0 \mathrm{~mm}$. Coronal reformats provided a good means to assess slice misregistration (stair-step artifact), blur, and anomalous signal loss (stripe artifact).

\section{Image Analysis}

All axial and coronally reformatted TRON, respiratory triggered, and free breathing diffusion-weighted images (at b-values of 50 and $500 \mathrm{~s} / \mathrm{mm}^{2}$ ) were independently reviewed by two board-certified radiologists (H.K.H. and P.S.L., with more than 10 and more than 5 years of clinical experience with MR imaging, respectively). Observers were blinded to the type of image under evaluation. Sharpness of vessels on both axial and coronally reformatted images was assessed using a five-point grading scale $(1=$ severe blurring, vessels not visible; $2=$ considerable blurring of vessels; $3=$ moderate blurring of vessels; $4=$ minimal blurring of vessels; $5=$ sharp vessels). On coronally reformatted images, so-called stair-step artifacts (i.e., stair-step-like deformation of organ contours, such as those of the liver and gallbladder) can be observed due to a mismatch between two sequential image acquisitions. Therefore, degree of stair-step artifacts was assessed using a five-point grading scale (1 = severe stair-step artifacts, $2=$ considerable stair-step artifacts; $3=$ moderate stair-step artifacts; $4=$ minimal stair-step artifacts; $5=$ no stair-step artifacts). Furthermore, so-called stripe artifacts can be observed in DWI of the liver. The exact cause of stripe artifacts has not been clarified yet, but they appear as horizontal stripes of variable signal intensity across axial slices viewed on coronally reformatted images. Therefore, degree of stripe artifacts was also assessed using a five-point grading scale $(1=$ severe stripe artifacts, $2=$ considerable stripe artifacts; $3=$ moderate

Table 1

DWI Single-Shot EPI Sequence Parameters

\begin{tabular}{lllc}
\hline \multicolumn{1}{c}{ DW ssh-EPI } & \multicolumn{1}{c}{ TRON } & \multicolumn{1}{c}{ Resp. Triggered } & Free breathing \\
\hline FOV $(\mathrm{mm})$ & $250-280 \times 245-330$ & $250 \times 280 \times 245-330$ & $250 \times 280 \times 245-330$ \\
Voxel (mm, acquired / reconstructed), matrix & $2.5 \times 2.5 \times 5 /$ & $2.5 \times 2.5 \times 5 /$ & $2.5 \times 2.5 \times 5 /$ \\
& $1.25 \times 1.25 \times 5$ & $1.25 \times 1.25 \times 5$ & $1.25 \times 1.25 \times 5$ \\
& $100-112 \times 98-130$ & $100-112 \times 98-130$ & $100-112 \times 98-130$ \\
Slice number, thickness/gap & $34 \times 5 \mathrm{~mm} / 0 \mathrm{~mm}$ & $34 \times 5 \mathrm{~mm} / 0 \mathrm{~mm}$ & $34 \times 5 \mathrm{~mm} / 0 \mathrm{~mm}$ \\
SENSE x & 2 & 2 & 2 \\
TR/TE (ms) & $4294 / 58$ & Matched to resp. cycle & $3691 / 58$ \\
EPI factor & $53-69$ & $53-69$ & $53-69$ \\
Averages, halfscan factor & $4,0.6$ & $4,0.6$ & $4,0.6$ \\
Fat saturation & SPIR & SPIR & SPIR \\
b-values (s/mm ${ }^{2}$ ) & $0,50,500$ & $0,50,500$ & $0,50,500$ \\
Nominal scan time (min:sec) & $02: 03-02: 06$ & $01: 45-01: 48$ & $01: 45-01: 48$ \\
\hline
\end{tabular}


Table 2

Comparison of Scores Regarding Sharpness of Vessels (Mean \pm SD) in TRON, Respiratory Triggered, and Free Breathing DWI*

\begin{tabular}{|c|c|c|c|}
\hline Slice orientation, b-value (b), and observer & TRON DWI & Respiratory triggered DWI & Free breathing DWI \\
\hline \multicolumn{4}{|l|}{ Axial } \\
\hline \multicolumn{4}{|l|}{$\mathrm{b}=50 \mathrm{~s} / \mathrm{mm}^{2}$} \\
\hline Observer 1 & $3.90 \pm 0.99\left(^{*}\right)$ & $4.30 \pm 0.82(\#)$ & $3.40 \pm 0.84\left(^{* \star}\right)$ \\
\hline Observer 2 & $4.40 \pm 0.70$ & $4.80 \pm 0.42(\#)$ & $3.90 \pm 1.10$ \\
\hline \multicolumn{4}{|l|}{$\mathrm{b}=500 \mathrm{~s} / \mathrm{mm}^{2}$} \\
\hline Observer 1 & $3.70 \pm 0.95\left(^{*}\right)$ & $4.30 \pm 0.82(\#)$ & $3.40 \pm 0.97$ \\
\hline Observer 2 & $3.50 \pm 0.97$ & $3.80 \pm 0.92(\#)$ & $2.90 \pm 1.29$ \\
\hline \multicolumn{4}{|l|}{ Coronal } \\
\hline \multicolumn{4}{|l|}{$\mathrm{b}=50 \mathrm{~s} / \mathrm{mm}^{2}$} \\
\hline Observer 1 & $3.60 \pm 0.84$ & $4.00 \pm 0.67(\#)$ & $3.10 \pm 0.74\left(^{* \star}\right)$ \\
\hline Observer 2 & $3.40 \pm 0.70\left(^{*}\right)$ & $4.10 \pm 0.88(\#)$ & $2.80 \pm 0.92\left(^{* *}\right)$ \\
\hline \multicolumn{4}{|l|}{$\mathrm{b}=500 \mathrm{~s} / \mathrm{mm}^{2}$} \\
\hline Observer 1 & $3.10 \pm 1.12\left(^{*}\right)$ & $3.60 \pm 0.70(\#)$ & $2.80 \pm 1.23$ \\
\hline Observer 2 & $2.90 \pm 0.88$ & $3.40 \pm 0.84(\#)$ & $2.30 \pm 0.68\left(^{* \star}\right)$ \\
\hline
\end{tabular}

${ }^{*}$ Significant difference $(P<0.05)$ of TRON versus respiratory triggered is labeled $(*)$, TRON versus free breathing $\left(^{\star \star}\right)$, respiratory triggered versus free breathing (\#). Scoring system: 1 = severe blurring, vessels not visible; $2=$ considerable blurring of vessels; $3=$ moderate blurring of vessels; $4=$ minimal blurring of vessels; $5=$ sharp vessels.

stripe artifacts; $4=$ minimal stripe artifacts; $5=$ no stripe artifacts).

\section{Statistical Analysis}

Mean actual scan times \pm SDs of TRON and respiratory triggered DWI relative to free breathing DWI (in $\%$ ) were calculated. Sharpness of vessels on axial and coronally reformatted images, degree of stair-step artifacts on coronally reformatted images, and degree of stripe artifacts on coronally reformatted images among TRON, respiratory triggered, and free breathing DWI (at b-values of 50 and $500 \mathrm{~s} / \mathrm{mm}^{2}$ ) were compared using Wilcoxon's signed rank tests. Differences were considered significant when $P$ values were less than 0.05. Statistical analyses were executed using Statistical Package for the Social Sciences version 15.0 software (SPSS, Chicago, IL).

\section{RESULTS}

Mean actual scan times \pm SDs of TRON and respiratory triggered DWI were $2 \min 22 \mathrm{~s} \pm 10.2 \mathrm{~s}$ (range, 2 min $8 \mathrm{~s}$ to $2 \min 42 \mathrm{~s}$ ) and $4 \mathrm{~min} 20 \mathrm{~s} \pm 43 \mathrm{~s}$ (range, $3 \mathrm{~min} 32 \mathrm{~s}$ to $6 \mathrm{~min} 10 \mathrm{~s})$. Mean scan time ratio \pm
SDs of TRON and respiratory triggered DWI relative to free breathing DWI were $1.34 \pm 0.10$ and $2.45 \pm$ 0.40 , respectively. Note, the actual scan time for free breathing DWI was the nominal scan time (1 min and 45-48 s), although it varied slightly with FOV.

Mean scores regarding sharpness of vessels are displayed in Table 2. In four of eight comparisons (two observers, two b-values, and two slice orientations), image sharpness in respiratory triggered DWI was significantly better $(P<0.05)$ than in TRON DWI. In four of eight comparisons, image sharpness in TRON DWI was significantly better $(P<0.05)$ than in free breathing DWI. Image sharpness in respiratory triggered DWI was always significantly better $(P<0.05)$ than in free breathing DWI.

Mean scores regarding degree of stair-step artifacts are displayed in Table 3. In two of four comparisons (two observers and two b-values), degree of stair-step artifacts in TRON DWI was significantly less $(P<$ 0.05) than in respiratory triggered DWI (Table 4). There were no significant differences in degree of stair-step artifacts between TRON and free breathing DWI (Table 4). In one of four comparisons, degree of stair-step artifacts in free breathing DWI was significantly less $(P<0.05)$ than in respiratory triggered DWI.

Table 3

Comparison of Scores Regarding Degree of Stair-Step Artifacts (Mean $\pm \mathrm{SD}$ ) and $P$ Values of Pairwise Comparisons (in Parenthesis) on Coronally Reformatted Images in TRON, Respiratory Triggered, and Free Breathing DWI*

\begin{tabular}{cccc}
\hline B-value $(\mathrm{b})$, and observer & TRON DWI & Respiratory triggered DWI & Free breathing DWI \\
\hline $\mathrm{b}=50 \mathrm{~s} / \mathrm{mm}^{2}$ & & & \\
$\quad$ Observer 1 & $3.70 \pm 0.48$ & $3.20 \pm 0.79$ & $3.30 \pm 0.95$ \\
$\quad$ Observer 2 & $3.70 \pm 0.82\left(^{*}\right)$ & $2.60 \pm 0.70(\#)$ & $3.60 \pm 0.84$ \\
$\mathrm{~b}=500$ s $/ \mathrm{mm}^{2}$ & & $3.10 \pm 0.74$ & $3.10 \pm 0.88$ \\
Observer 1 & $3.50 \pm 0.53$ & $2.30 \pm 0.82$ & $2.80 \pm 0.92$ \\
Observer 2 & $3.40 \pm 0.52\left(^{*}\right)$ & \\
\hline
\end{tabular}

*Significant difference $(P<0.05)$ of TRON versus respiratory triggered is labeled $\left.{ }^{*}\right)$, TRON versus free breathing $\left(^{* *}\right)$, or respiratory triggered versus free breathing (\#). Scoring system: $1=$ severe stair-step artifacts, $2=$ considerable stair-step artifacts; $3=$ moderate stairstep artifacts; $4=$ minimal stair-step artifacts; $5=$ no stair-step artifacts. 
Table 4

Comparison of scores regarding degree of stripe artifacts (mean \pm SD) on coronally reformatted images in TRON, respiratory triggered, and free breathing $\mathrm{DWI}{ }^{*}$

\begin{tabular}{cccc}
\hline B-value $(b)$, and observer & TRON DWI & Respiratory triggered DWI & Free breathing DWI \\
\hline $\mathrm{b}=50 \mathrm{~s} / \mathrm{mm}^{2}$ & & & $3.60 \pm 0.70(\#)$ \\
$\quad$ Observer 1 & $3.50 \pm 0.71$ & $3.50 \pm 0.85$ & $3.00 \pm 0.47$ \\
Observer 2 & $3.40 \pm 0.52$ & & $3.10 \pm 0.32$ \\
$\mathrm{~b}=500 \mathrm{~s} / \mathrm{mm}^{2}$ & & $2.90 \pm 0.57$ & $2.50 \pm 0.85$ \\
Observer 1 & $2.40 \pm 0.70$ & $2.50 \pm 0.85$ & $2.30 \pm 0.82$ \\
Observer 2 & $2.50 \pm 0.97$ &
\end{tabular}

${ }^{*}$ Significant difference $(\mathrm{p}<0.05)$ of TRON versus respiratory triggered is labeled $\left({ }^{*}\right)$, TRON versus free breathing $\left.{ }^{* *}\right)$, respiratory triggered versus free breathing (\#). Scoring system: 1 = severe stripe artifacts, $2=$ considerable stripe artifacts; $3=$ moderate stripe artifacts; $4=$ minimal stripe artifacts; $5=$ no stripe artifacts.

Mean scores regarding degree of stripe artifacts are displayed in Table 4 . There were no significant differences in degree of stripe artifacts between TRON and respiratory triggered DWI, and between TRON and free breathing DWI. In one of four comparisons (two observers and two b-values), degree of stripe artifacts in respiratory triggered DWI was significantly less $(P<0.05)$ than in free breathing DWI.

Figure 3 shows a representative example of a part of the navigator profile in TRON using the maximum displacement threshold set to $10 \mathrm{~mm}$, and Figure 4 shows a representative example of TRON DWI, com- pared with respiratory triggered and free breathing DWI.

\section{DISCUSSION}

DWI is an emerging technique for the detection of liver lesions due to its high lesion-to-background contrast (1,6-10). Compared with breathhold DWI, respiratory triggered (either using a conventional navigator echo technique or respiratory triggering) and free breathing DWI have the advantage of increasing SNR by

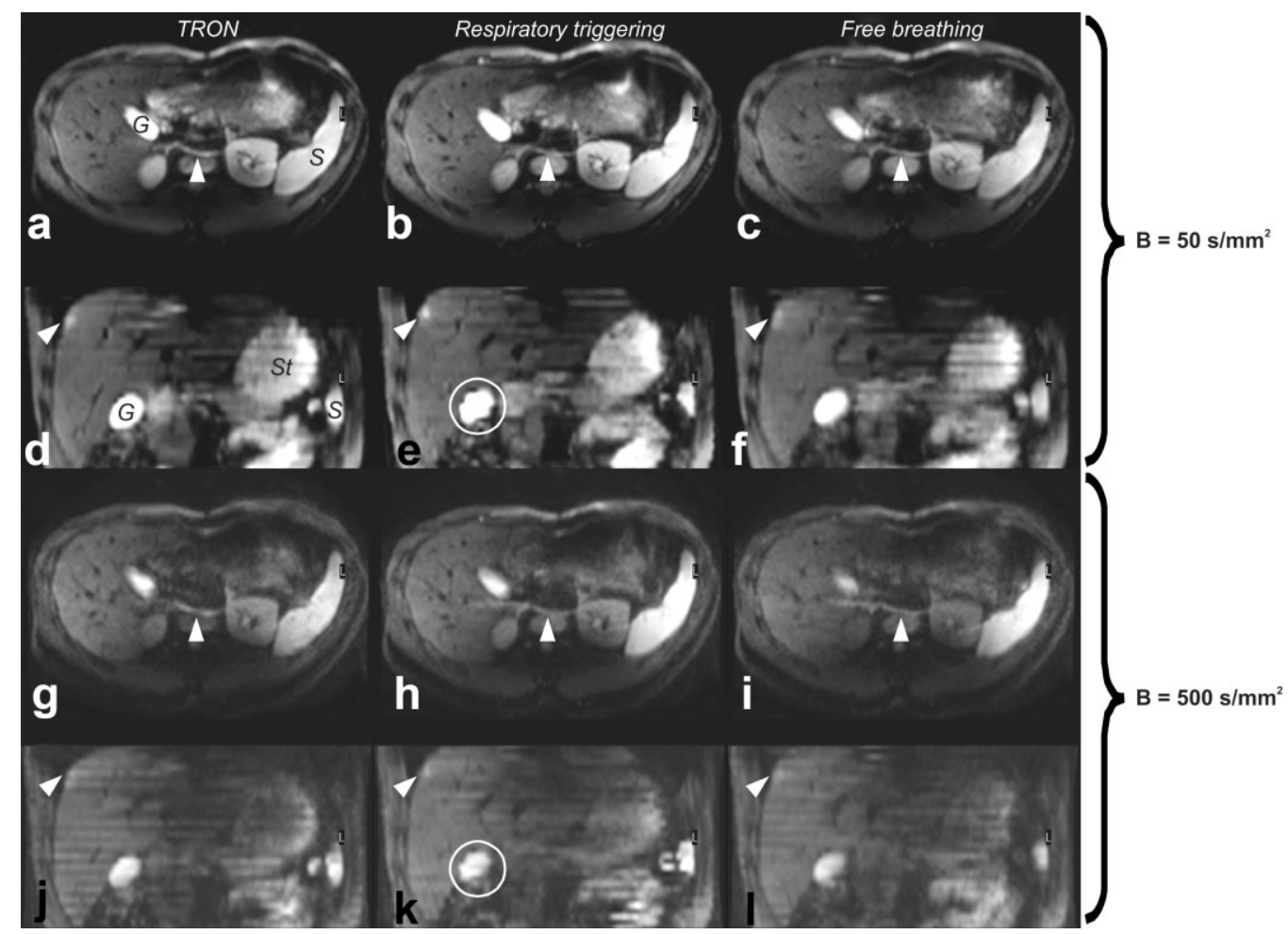

Figure 4. Representative example of TRON DWI (a,d,g,j), compared with respiratory triggered (b,e,h,k), and free breathing DWI $(\mathbf{c}, \mathbf{f}, \mathbf{i}, \mathbf{1})$, at b-values of $50 \mathrm{~s} / \mathrm{mm}^{2}$ and $500 \mathrm{~s} / \mathrm{mm}^{2}$, in a 28-year-old healthy male volunteer. Axial images (a-c and g-i), coronally reformatted images ( $\mathrm{d}-\mathrm{f}$ and $\mathrm{j}-\mathrm{k}$ ), " $G$ " denotes the gallbladder, " $S$ " denotes the spleen, and "St" denotes the stomach. On both axial and coronally reformatted images, sharpness of vessels is better in TRON DWI (a,d,g,j) and respiratory triggered DWI (b,e,h,k), compared with free breathing DWI (c,f,i,l). Note stair-step artifacts in coronally reformatted respiratory triggered DWI, for example in the gallbladder (encircled), which are due to inappropriate timing of the trigger events. Also note stripe artifacts of the liver on all coronally reformatted images of both TRON, respiratory triggered, and free breathing DWI, which appear to be more severe at a b-value of $500 \mathrm{~s} / \mathrm{mm}^{2}$ (j-l) than at a b-value of $50 \mathrm{~s} / \mathrm{mm}^{2}$ (d-f). Also note EPI fat shift artifacts from insufficiently suppressed fat (arrowheads). 
acquiring multiple signal averages, which allows acquisition of thinner slices. This, in turn, may increase lesion detectability of DWI (1). However, respiratory triggered DWI suffers from a two-to three-fold increased scan time (compared with free breathing DWI) $(1,2)$. Furthermore, respiratory triggering potentially suffers from unexpected severe scan time prolongation if respiration becomes irregular or elongated by apnea due to the patient's deep sleep. Thus, patient throughput in respiratory triggered DWI is relatively low. On the other hand, free breathing DWI while being very time-efficient, suffers from image blurring thereby decreasing lesion conspicuity and lesion delineation.

The recently introduced TRON technique allows for continuous real-time slice tracking and position correction, without using any gating window, thereby overcoming the disadvantages of both respiratory triggered and free breathing DWI (2-4). By exploiting the higher SNR at 3.OT, lesion-to-background contrast and liver lesion detectability at DWI may be increased compared with DWI at $1.5 \mathrm{~T}(5,11)$. However, it was still unknown whether DWI at 3.0T could be combined with TRON. The results of the present study showed that it is feasible to combine DWI at 3.OT with TRON. Despite the addition of the maximum displacement threshold to this sequence, scan time was only modestly prolonged relative to free breathing DWI (34\% on average), which was considerably less compared with relative scan time prolongation in respiratory triggered DWI (145\% on average). Furthermore, image sharpness in TRON DWI was significantly better $(P<0.05)$ than in free breathing DWI in four of eight comparisons (two observers, two bvalues, and two slice orientations). However, image sharpness in respiratory triggered DWI was significantly better $(P<0.05)$ than in TRON DWI in four of eight comparisons. This is probably due to the fact that maximum displacement was set to $10 \mathrm{~mm}$, and tracking errors of less than $10 \mathrm{~mm}$ may have reduced image sharpness. However, reducing the maximum displacement would prolong scan time, because more data would be discarded. On the other hand, degree of stair-step artifacts in TRON DWI was significantly less $(P<0.05)$ than in respiratory triggered DWI in two of four comparisons. Stair-step artifacts in respiratory triggered DWI are caused by inappropriate timing of trigger events (Fig. 4). Stair-step artifacts may reduce reliability of volume or apparent diffusion coefficient (ADC) measurements in DWI for the assessment of response to therapy. In this respect, TRON may be a good alternative to respiratory triggered DWI. On coronally reformatted images stripe artifacts were observed in both TRON, respiratory triggered, and free breathing DWI, without any significant differences among the three DWI sequences (except in one of four comparisons between respiratory triggered and free breathing DWI). However, degree of stripe artifacts appeared to be more severe at a b-value of $500 \mathrm{~s} / \mathrm{mm}^{2}$ than at a b-value of $50 \mathrm{~s} /$ $\mathrm{mm}^{2}$ (Fig. 4). A possible explanation of the stripe artifact may be the so-called hepatic pseudo-anisotropy artifact (12). The hepatic pseudo-anisotropy artifact refers to localized signal loss in the liver parenchyma at DWI, depending on the MPG direction. This artifact is probably caused by intravoxel deformation and/or acceleration of the liver during the period in which the MPGs are applied (12). It was speculated that this phenomenon mainly occurs at the end of expiration (12), although this has not yet been confirmed. When using interleaved slice ordering, either the even or odd slices may be affected by the hepatic pseudo-anisotropy artifact and consequent signal loss, which results in the characteristic stripe artifacts seen on coronally reformatted images. Furthermore, this phenomenon may become more pronounced when using heavier diffusion-weighting, which explains the difference in degree of stripe artifacts between the images obtained with a b-value of $50 \mathrm{~s} / \mathrm{mm}^{2}$ and those obtained with a b-value of $500 \mathrm{~s} / \mathrm{mm}^{2}$. Because stripe artifacts may interfere with lesion detectability and $\mathrm{ADC}$ measurements, further investigation is necessary to solve this issue.

A limitation of the present study is that only healthy volunteers were included and TRON has not proven yet to be able to detect more lesions than respiratory gated or free breathing DWI. In addition, it is still unknown whether ADC measurements of liver lesions in TRON DWI are accurate, although we have no a priori reason to assume that they would be less reliable than in respiratory gated or free breathing DWI. Therefore, future patient studies are necessary. Another issue is that short inversion time inversion recovery (STIR) fat suppression may be preferred for DWI of the liver at 3.0T, instead of SPIR fat suppression (Fig. 4), because the former gives superior global fat suppression over an extended field of view and is insensitive to magnetic field inhomogeneities (13). STIR, however, is not appropriate for use in combination with the navigator echo in TRON.

In conclusion, DWI of the liver at 3.0T using TRON is feasible. Image sharpness in TRON DWI is superior to that in free breathing DWI. Although image sharpness of respiratory triggered DWI is still better, TRON DWI requires less scan time and reduces stair-step artifacts compared with respiratory triggered DWI.

\section{ACKNOWLEDGMENT}

The authors thank Jouke Smink for advice and discussion in reviewing this article.

\section{REFERENCES}

1. Naganawa S, Kawai H, Fukatsu H, et al. Diffusion-weighted imaging of the liver: technical challenges and prospects for the future. Magn Reson Med Sci 2005;4:175-186.

2. Takahara T, Ogino T, Okuaki T, et al. Respiratory gated body diffusion weighted imaging avoiding prolongation of scan time: tracking only navigator echo (TRON) technique. In: Presentation of the 16th Joint Annual Meeting of ISMRM-ESMRMB, Berlin, Germany, May 19-25, 2007.

3. Takahara T, Imai Y, Yamashita T, Yasuda S, Nasu S, Van Cauteren M. Diffusion weighted whole body imaging with background body signal suppression (DWIBS): technical improvement using free breathing, STIR and high resolution 3D display. Radiat Med 2004;22:275-282. 
4. Kwee TC, Takahara T, Ochiai R, Nievelstein RA, Luijten PR. Diffusion-weighted whole-body imaging with background body signal suppression (DWIBS): features and potential applications in oncology. Eur Radiol 2008;18:1937-1952.

5. Schick F. Whole-body MRI at high field: technical limits and clinical potential. Eur Radiol 2005;15:946-959.

6. Xu PJ, Yan FH, Wang JH, Lin J, Ji Y. Added value of breath hold diffusion-weighted MRI in detection of small hepatocellular carcinoma lesions compared with dynamic contrast-enhanced MRI alone using receiver operating characteristic curve analysis. J Magn Reson Imaging 2009;29:341-349.

7. Goshima S, Kanematsu M, Kondo H, et al. Diffusion-weighted imaging of the liver: optimizing $b$ value for the detection and characterization of benign and malignant hepatic lesions. J Magn Reson Imaging 2008;28:691-697.

8. Zech CJ, Herrmann KA, Dietrich O, Horger W, Reiser MF, Schoenberg SO. Black-blood diffusion-weighted EPI acquisition of the liver with parallel imaging: comparison with a standard T2weighted sequence for detection of focal liver lesions. Invest Radiol 2008;43:261-266.
9. Parikh T, Drew SJ, Lee VS, et al. Focal liver lesion detection and characterization with diffusion-weighted MR imaging: comparison with standard breath-hold T2-weighted imaging. Radiology 2008; 246:812-822.

10. Coenegrachts K, Delanote J, Ter Beek L, et al. Improved focal liver lesion detection: comparison of single-shot diffusionweighted echoplanar and single-shot T2 weighted turbo spin echo techniques. Br J Radiol 2007;80:524-531.

11. Van den Bos IC, Hussain SM, Krestin GP, Wielopolski PA. Liver imaging at 3.0T: diffusion-induced black-blood echoplanar imaging with large anatomic volumetric coverage as an alternative for specific absorption rate-intensive echo-train spin-echo sequences: feasibility study. Radiology 2008;248: 264-271.

12. Nasu K, Kuroki Y, Fujii H, Minami M. Hepatic pseudo-anisotropy: a specific artifact in hepatic diffusion-weighted images obtained with respiratory triggering. MAGMA 2007;20:205-211.

13. Delfaut EM, Beltran J, Johnson G, Rousseau J, Marchandise X, Cotten A. Fat suppression in MR imaging: techniques and pitfalls. Radiographics 1999;19:373-382. 